\title{
$k$-INVARIANTS IN LOCAL COEFFICIENT THEORY
}

\author{
JERROLD SIEGEL ${ }^{1}$
}

\begin{abstract}
A transgression-obstruction theorem is presented for not necessarily simply connected spaces. This theorem is used to produce an explicit model for a classifying space for fibre homotopy equivalence classes of fibrations with fibre a $K(\pi, n)$.
\end{abstract}

This note has two purposes. The first is to complete the general treatment of obstruction theory begun in [7]. This theory does not make the usual assumption about trivial action of the fundamental group on the higher homotopy groups, yet it does make available most of the geometric tools usually associated with the "1-connected theory." The major defect in the theory presented in [7] was the ad hoc definition of $k$-invariants used there. The definition made necessary some special arguments which threw little light on the general theory. What we overlooked was the fact that using the tools developed in [7] we could prove a straightforward generalization of the transgression-obstruction theorem, hence, making possible a convenient algebraic definition of the $k$-invariants. In this note we exhibit this theorem. This theorem is, in fact, a special case of a more general theorem of J. F. McClendon [6]. We thank the referee for pointing this out.

An application of the transgression-obstruction theorem will be given in the second section. We construct an explicit model of a classifying space for fibre homotopy equivalence classes of fibrations with fibre $K(\pi, n)$. The existence of such a space is known from general considerations (see, for example, [3]); however, there considerations do not lead to a clear understanding of the nature of the classifying space. It is hoped that the more complete description of this classifying space presented below will lead to some sort of decomposition theory for classifying spaces of more general fibres.

1. Moore-Postnikov theory. In this section we give a treatment of the central technical lemma of Moore-Postnikov theory without ref-

Received by the editors December 22, 1969.

AMS 1970 subject classifications. Primary 55F35, 55G45.

Key words and phrases. Obstruction theory, Moore-Postnikov theory, $k$-invariants, local coefficient theory, transgression-obstruction theorem, fibre homotopy equivalence, classifying space, $K(\pi, n)$.

1 During the writing of this paper the author was supported in part by NSF Grant GP-6955.

Copyright @ 1971, American Mathematical Society 
erence to the fundamental group of the space involved. Naturally, it is necessary to consider the action of the fundamental group. This is accomplished by considering our $k$-invariants as being in a cohomology group with an appropriate local coefficient system. We make use of the spectral sequence defined in [7]. We adopt the terminology and notation of that paper.

1.1. Notation. Let $F \subseteq E \stackrel{p}{\rightarrow} B$ be a fibre space with $\pi_{k}(F)=0$, $k \leqq n-1$ and $n \geqq 2$. Let $J_{n}$ be the system of groups $\left\{\pi_{n}(F)\right\}$ over $B$. Let $\mathfrak{H}^{q}\left(F, \mathfrak{J}_{n}\right)$ be the coefficient system over $B$ based on $H^{q}\left(F, \pi_{n}(F)\right)$. Finally, let $[i] \in H^{n}\left(F, \pi_{n}(F)\right)$ be the fundamental class.

1.2. TheOREM. Let $F \subseteq E \stackrel{p}{\rightarrow} B$ be as in 1.1. There is a spectral sequence

$$
E_{r}^{p, q} \Longrightarrow H^{*}\left(E, p^{*} \jmath_{n}\right)
$$

with $E_{2}^{p, q} \simeq H^{p}\left(B, \mathfrak{H}^{q}\left(F, \mathfrak{J}_{n}\right)\right)$.

Proof. [7].

1.3. TheOREM. In the spectral sequence of $1.2,[i]$ transgresses to a class we call $\tau[i] \in H^{n+1}\left(B, J_{n}\right)$.

Proof. By 2.12 of [7] we know $\delta_{1}^{*}[i]=0$ iff $[i]$ is invariant under the action of $\pi_{1}\left(B, b_{0}\right)$. The system of groups $J_{n}$ is precisely that which is required to give this condition in $\mathcal{F}^{n}\left(F, J_{n}\right)$. The remainder of the necessary differentials vanish since they hit the 0-group.

The class $\tau[i]$ is essentially the $k$-invariant of the first stage of a Postnikov decomposition of $F \subseteq E \stackrel{p}{\rightarrow} B$. However, first we must review some geometric constructions presented in [7].

1.4. Review of [7]. (a) Let $G$ be a group. Let $\left(X, x_{0}\right)$ be a basepointed space with a base point fixed action of $G$ on $X$. There are obvious $G$-actions on $P\left(X, x_{0}\right)$ (the space of paths starting at $\left.x_{0}\right)$ and $\Omega\left(X, x_{0}\right)$ (the space of loops). Moreover, the fibre map $e: P\left(X, x_{0}\right) \rightarrow X$ and the pairing

$$
\mu: \Omega\left(X, x_{0}\right) \times P\left(X, x_{0}\right) \rightarrow P\left(X, x_{0}\right)
$$

are equivariant with respect to these actions.

(b) Let $\xi \rightarrow Y$ be a principal $G$-bundle, let $\xi(X) \stackrel{\prime}{\rightarrow} Y$ be the induced bundle with fibre $X$. We have the following commutative diagram:

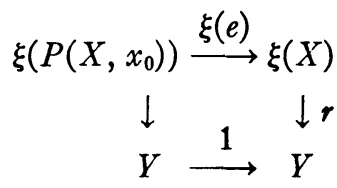


with $\xi(e)$ a Serre fibration [5].

Suppose we have a map $w: B \rightarrow \xi(X)$. We have a fibration $p$ induced by the diagram

$$
\begin{array}{ccc}
\xi(w, X) \stackrel{\tau}{\rightarrow} & \xi\left(P\left(X, x_{0}\right)\right) \\
p \downarrow & & \downarrow \xi(e) \\
B & \stackrel{w}{\rightarrow} & \xi(X)
\end{array}
$$

If we have a base point $b_{0}$ for $B$ and if $w\left(b_{0}\right)=x_{0}$ we have the fibre of $p$ over $b_{0}$ as $\Omega\left(X, x_{0}\right)$. Finally, if $\left((r w)^{*} \xi\left(\Omega\left(X, x_{0}\right)\right)\right) \Delta \xi(w, X)$ is the Whitney product, $\mu$ induces

$$
\tilde{\mu}:\left((r w)^{*} \xi\left(\Omega\left(X, x_{0}\right)\right)\right) \Delta \xi(w, X) \rightarrow \xi(w, X) .
$$

1.5. Notation. Below we will be interested in the case where $Y$ is a $K\left(\pi_{1}, 1\right)$ and $\xi$ is $\hat{\pi}_{1}$ the universal bundle. We will let $X$ be a $K(\pi, n+1)$, we will let $\Phi$ be the action of $\pi_{1}$ on $K(\pi, n+1)$ (see $[7$, p. 2$])$. We denote the space $\hat{\pi}(K(\pi, n+1))$ by $L_{\pi_{1}}(\Phi, n+1)$. We remark that if $f: B \rightarrow K\left(\pi_{1}, 1\right)$ is fixed, then the group of homotopy classes of liftings

$$
\begin{aligned}
& L_{\pi_{1}}(\Phi, n+1) \\
& w \nearrow \downarrow r \\
& B \underset{f}{\rightarrow} K\left(\pi_{1}, 1\right)
\end{aligned}
$$

is isomorphic to $H^{n+1}\left(B, \Phi\left(f^{*} \hat{\pi}\right)\right)$ (see $[5$, p. 2]). We denote $\hat{\pi}(w, K(\pi, n+1))$ by $E_{w}$.

1.6. TheOREM. Assume we are in the situation of 1.1. Let $w: B$ $\rightarrow L_{\pi_{1}}(\Phi, n+1)$ represent $-\tau([i])$. Consider the fibration $K(\pi, n)$ $\subseteq E_{w} \stackrel{p}{\rightarrow} B$. There is a map, $t$, that makes the following diagram commute.

$$
\begin{array}{cl}
E \stackrel{t}{\rightarrow} E_{w} \\
p \downarrow & \quad \downarrow p \\
B= & B
\end{array}
$$

Moreover if $v=t / F$ then $v: F \rightarrow K(\pi, n)$ represents the class of $[i]$.

Proof. [6].

The proof is a formal generalization of the usual one.

2. $B(K(\pi, n)), \pi$ abelian. In this section we show that, with a proper choice of $\pi_{1}$ and $\Phi$, the space $L_{\pi_{1}}(\Phi, n+1)$ is a classifying space for fibre homotopy equivalence classes of fibrations with fibre 
$K(\pi, n)$. We then study the homology of this space using the tools developed in [8].

It should be noted that the notion of classifying space is modified in that we consider base point free maps and homotopies (see [1], [3], [9]).

We begin this section with a notion of fibre homotopy equivalence which corresponds to the base point fixed point case.

2.1. Definition. (a) A fibration $E \stackrel{p}{\rightarrow}\left(B, b_{0}\right)$ with fibre $F$ is a fibre space with fibre $p^{-1}\left(b_{0}\right)$ the homotopy type of $F$ ( $F$ fixed) and with a fixed homotopy equivalence $h: F \rightarrow p^{-1}\left(b_{0}\right)$ we denote any homotopy inverse of $h$ as $h^{-1}$.

(b) By the fibre homotopy equivalence $f$, between $E \stackrel{p}{\rightarrow}\left(B, b_{0}\right)$ and $E^{\prime} \stackrel{p^{\prime}}{\rightarrow}\left(B, b_{0}\right)$ both with fibre $F$ we mean the usual thing without reference to $F$. We write $f: E \simeq E^{\prime}$.

(c) A strong fibre homotopy equivalence between $E \stackrel{p}{\rightarrow}\left(B, b_{0}\right)$ and $E^{\prime} \stackrel{p^{\prime}}{\rightarrow}\left(B, b_{0}\right)$ is a fibre homotopy equivalence

$$
\begin{aligned}
& \begin{array}{c}
E \stackrel{f}{\simeq} \underset{E^{\prime}}{\simeq} \underset{\downarrow p^{\prime}}{ }
\end{array} \\
& B=B
\end{aligned}
$$

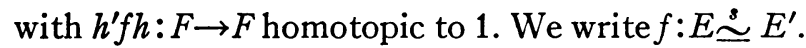

We now consider the following situation. Let $A(\pi)$ be the group of automorphism of $\pi$. Let $\Phi: A(\pi) \times \pi \rightarrow \pi$ be the obvious action. We consider $L_{A(\pi)}(\Phi, n+1)$.

2.2. ThEOREM. The space $L_{A(\pi)}(\Phi, n+1)$ is a classifying space for strong fibre homotopy equivalence classes of fibrations with fibre $K(\pi, n)$. We take our maps and homotopy classes of maps as base point fixed.

Proof. Given any action $\Phi_{1}: G \times \pi \rightarrow \pi$ we have an induced homomorphism $\hat{h}: G \rightarrow A(\pi)$. This homomorphism in turn induces the following diagram.

$$
\begin{gathered}
L_{G}\left(\Phi_{1}, n+1\right) \stackrel{\bar{h}}{\rightarrow} L_{A(\pi)}(\Phi, n+1) \\
p \downarrow \\
K(G, 1) \stackrel{h}{\rightarrow} K(A(\pi), 1) .
\end{gathered}
$$

Given a fibration $K(\pi, n) \subseteq E \rightarrow B$, let $G=\pi_{1}\left(B, b_{0}\right)$ and $\Phi_{1}: G \times \pi \rightarrow \pi$, the action of the fundamental group on $\pi$. Theorem 1.6 tells us that there is a map $w: B \rightarrow L_{G}\left(\Phi_{1}, n+1\right)$ with $[w]=-\tau[i]$ and so that $K(\pi, n) \subseteq E_{w} \stackrel{p}{\rightarrow} B$ is strongly fibre homotopy equivalent to $K(\pi, n)$ 
$\subseteq E \stackrel{p}{\rightarrow} B$. Notice the implicit use of Dold's theorem [2, 6.1]. Also implicit is the fact that up to strong fibre homotopy equivalence $E_{w}$ is independent of $w \in[w]$. We now map the class of $K(\pi, n) \subseteq E \rightarrow B$ into $[\bar{h} w] \in\left[B, L_{A(\pi)}(\Phi, n+1)\right]_{b p}$. It is clear that this map is well defined since if

$$
\begin{aligned}
& E \stackrel{f}{\longrightarrow} E^{\prime} \\
& \downarrow \\
& B=B
\end{aligned}
$$

is a strong homotopy equivalence then $f^{*}\left[i^{\prime}\right]=[i]$; hence $\tau\left[i^{\prime}\right]=\tau[i]$. Notice $E_{\overline{h_{0}}} \stackrel{\stackrel{s}{\underline{s}}}{\underline{E_{w}}}$.

It is also clear that the map is onto since if $[\bar{h} w] \in\left[B, L_{A(\pi)}(\Phi, n+1)\right]_{b p}$, $K(\pi, n) \subseteq E_{w} \rightarrow B$ maps onto $[\bar{h} w]$. To show the map is one-one we

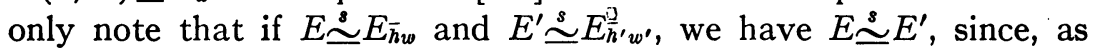

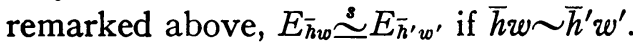

2.3. TheOREM. The space $L_{\mathbf{A}(x)}(\Phi, n+1)$ is a classifying space for fibre homotopy equivalence classes of fibration with fibre $K(\pi, n)$. We take our maps and homotopy classes of maps base point free.

Proof. We can appeal to Dold [3, p. 16.8]. Or we can prove the result directly by noting that if $E \simeq E^{\prime}$ is a fibre homotopy equivalence, $h^{\prime} f h: K(\pi, n) \rightarrow K(\pi, n)$ corresponds to an automorphism $A \in \operatorname{Aut}(\pi)$; moreover $\hat{h}$ and $\hat{h}^{\prime}: G \rightarrow \operatorname{Aut}(\pi)$ are related by the formula $\hat{h}^{\prime}(g)=A \hat{h}(g) A^{-1} \cdot \tau[i]$ and $\tau\left[i^{\prime}\right]$ are related similarly. One now checks this is precisely the effect of a free homotopy going around a closed loop in $L_{\boldsymbol{A}(x)}(\Phi, n+1)$ representing $A$.

We now study the homology of $L_{A(\pi)}(\Phi, n+1)$. We will be able to describe it in terms of more familiar algebraic objects.

Let us suppose that we have chosen a "free" $A(\pi)$ model for $K(\pi, n)$. That is, suppose $A(\pi)$ acts freely on the cells of $K(\pi, n)$ except in dimension 0 where there is a single 0 -cell. Let $C_{*}(K(\pi, n), Z)$ be the cell chain complex of this model. Let $\bar{C}_{*}(K(\pi, n), Z)$ $\subseteq C_{*}(K(\pi, n), Z)$ be the subcomplex generated by elements of the form $[C]-A[C] A \in A(\pi)$. Define

$$
\tilde{C}_{*}(K(\pi, n), Z)=C_{*}(K(\pi, n), Z) / \bar{C}_{*}(K(\pi, n), Z) .
$$

\subsection{THEOREM.}

$$
H_{q}\left(L_{A(\pi)}(\Phi, n+1), Z\right) \simeq H_{q}\left(\tilde{C}_{*}(K(\pi, n), Z)\right) \oplus H_{q}(K(A(\pi), 1), Z) .
$$

Proof. This is the homology version of 3.7 of [8] with projective in place of injective. 
2.6. Final remarks. The cohomology can be derived by the universal coefficient theorem; however it is not clear that a general version of 2.5 exists. [8] tells us that, for example, $Z$ is not a good ring for the particular cohomology spectral sequence used. More important, the consideration of $[8]$ is based on the possibility that $A(\pi)$ is finite; this is not in general present. In fact, a little thought tells us that there should be a difference between equivariant homology and cohomology for infinite groups of action.

The case $n=1, \pi$ nonabelian, is not covered in our theory for obvious reasons; however Gottlieb [4] has discovered a good deal of information about this case.

\section{BIBLIOGRAPHY}

1. G. Allaud, On the classification of fibre spaces, Math. Z. 92 (1966), 110-125. MR $32 \# 6462$.

2. A. Dold, Partitions of unity in the theory of fibrations, Ann. of Math. (2) 78 (1963), 223-255. MR 27 \#5264.

3. - Halbexakte Homotopiefunktoren, Lecture Notes in Math., no. 12, Springer-Verlag, Berlin, 1966. MR 33 \#6622.

4. D. H. Gottlieb, On fibre spaces and the evaluation map, Ann. of Math. (2) 87 (1968), 42-55. MR $36 \# 4560$.

5. W. Huebsch, On the covering homotopy theorem, Ann. of Math. (2) 61 (1955), 555-563. MR 19, 974.

6. J. F. McClendon, Obstruction theory in fibre spaces, Math. Z. (to appear).

7. J. Siegel, Higher order cohomology operations in local coefficient theory, Amer. J. Math. 89 (1967), 909-931. MR 37 \#913.

8. - Cohomology operations in local coefficient theory, Illinois J. Math. 15 (1971), 52-63.

9. J. Stasheff, A classification theorem for fibre spaces, Topology 2 (1963), 239-246. MR 27 \#4235.

Purde University, Lafayette, INdiana 47907 